\title{
Steroid therapy in adrenal insufficiency
}

\begin{abstract}
Adrenal insufficiency (AI) is a condition arising due to inadequate amounts of circulating steroid hormones, primarily cortisol; but may also include impaired production of aldosterone (a mineralocorticoid), which regulates sodium conservation, potassium secretion, and water retention. It occurs due to adrenal gland dysfunction, and primary management is corticosteroid supplementation, however, there is no universally accepted regimen for such replacement therapy. High doses and long duration of steroid coverage, that have traditionally been used, have not been able to reflect the physiological hypothalamicpituitary-adrenal response; hence control of disease symptomatology is not adequately achieved. We discuss few recommendations targeting such concerns, with emphasis on current replacement strategies.
\end{abstract}

Keywords: adrenal insufficiency, corticosteroids, replacement
Volume 6 Issue 2 - 2017

\author{
Ayan Mukherjee, Lahiry Sandeep, Shouvik \\ Choudhury, Dwaipayan Sarathi Chakraborty, \\ Sinha Rajasree \\ Department of Pharmacology, Institute of Post Graduate \\ Medical Education and Research, India
}

Correspondence: Sandeep Lahiry, Department of Pharmacology, Institute of Post Graduate Medical Education and Research, 244 B A.J.C Bose Road, Kolkata, India, Email sndplry@gmail.com

Received: August 24, 2017 | Published: September 25, 2017

\section{Introduction}

Hypoadrenalism or Adrenal Insufficiency (AI) can be classified as:

\section{Primary adrenal insufficiency (PAI)}

A condition which is due to impairment of the adrenal glands. The causes include:

Addison's disease or autoimmune adrenalitis: These are autoimmune conditions, constituting $80 \%$ of all cases. Autoimmune adrenalitis may be part of Type 2 autoimmune polyglandular syndrome, which can include type 1 diabetes, hyperthyroidism, and autoimmune thyroid disease (also known as Hashimoto's thyroiditis), and Hashimoto's disease. Hypogonadism may also present with this syndrome. Other diseases that are more common in people with autoimmune adrenalitis include premature ovarian failure, celiac disease, and autoimmune gastritis with pernicious anemia. Other subtypes include idiopathic (unknown) cause, congenital adrenal hyperplasia or an adenoma (tumor) of the adrenal gland.

Secondary adrenal insufficiency: A condition which is caused by impairment of the pituitary gland or hypothalamus. Its principal causes include pituitary adenoma (which can suppress production of adrenocorticotropic hormone (ACTH) and lead to adrenal deficiency unless the endogenous hormones are replaced); and Sheehan's syndrome, which is associated with impairment of only the pituitary gland. Tertiary adrenal insufficiency: A condition which is due to hypothalamic disease and reduction in the release of corticotropin releasing hormone $(\mathrm{CRH})$. Causes can include brain tumors and sudden withdrawal from long-term exogenous steroid use (which is the most common cause overall). ${ }^{1-3}$

For accurate diagnosis, it is first important to understand the exact pathophysiology behind AI. Low levels of glucocorticoids leads to systemic hypotension (one of the effects of cortisol is to increase peripheral resistance), which results in a decrease in stretch of the arterial baroreceptors of the carotid sinus and the aortic arch. This removes the tonic vagal and glossopharyngeal inhibition on the central release of ADH: high levels of ADH will ensue, which will subsequently lead to increase in water retention and hyponatremia. In contrast to mineralocorticoid deficiency, glucocorticoid deficiency does not cause a negative sodium balance (in fact a positive sodium balance may occur) ${ }^{4-6}$
$\mathrm{AI}$ is also classified according to the development and progression of the disease, i.e. acute and chronic AI. Causes of acute AI are mainly sudden withdrawal of long-term corticosteroid supplementation therapy (Waterhouse-Friderichsen syndrome), and related stress in people with underlying chronic AI (critical illness-related corticosteroid insufficiency). ${ }^{1,2}$ It is because, the use of high-dose steroids for more than a week begins to produce suppression of the person's adrenal glands because the exogenous glucocorticoids suppress hypothalamic CRH and pituitary ACTH. With prolonged suppression, the adrenal glands atrophy (physically shrink), and can take months to recover full function after discontinuation of the exogenous glucocorticoid. During this recovery time, the person is vulnerable to AI during times of stress, such as illness, due to both adrenal atrophy and suppression of $\mathrm{CRH}$ and ACTH release. Use of steroids joint injections may also result in adrenal suppression after discontinuation.

For chronic AI, the major contributors are autoimmune adrenalitis (Addison's Disease), tuberculosis, AIDS, and metastatic disease. Minor causes include systemic amyloidosis, fungal infections, hemochromatosis, and sarcoidosis. In rare occasions, Adrenoleukodystrophy can also cause AI..$^{1-3}$

\section{Sympatomatology}

Signs and symptoms of AI develop gradually and insidiously and include: hypoglycemia, dehydration, weight loss, disorientation, weakness, tiredness, dizziness, orthostatic hypotension, cardiovascular collapse, muscle aches, nausea, vomiting, and diarrhea. There may be associated tanning of the skin with systemic dermal patches. Characteristic sites of tanning are skin creases (e.g. of the hands) and the inside of the cheek (buccal mucosa). Goitre and vitiligo may also be present. ${ }^{1-3}$

\section{Diagnosis}

The best diagnostic tool to confirm adrenal insufficiency is the ACTH $(250 \mu \mathrm{g})$ stimulation test; however, if a patient is suspected to be suffering from an acute adrenal crisis, immediate treatment with parenetral corticosteroids is imperative and should not be delayed for any testing, as the patient's health can deteriorate rapidly and result in death without replacing the corticosteroids. Usually, Dexamethasone is used as the corticosteroid if the plan is to do the ACTH $(250 \mu \mathrm{g})$ stimulation test at a later time as it is the only corticosteroid that will not affect the test results. 
If not performed during crisis, then tests must include: random cortisol, serum ACTH, aldosterone, renin, potassium and sodium. A CT of the adrenal glands should be used to check for structural abnormalities of the adrenal glands. An MRI of the pituitary can be used to check for structural abnormalities of the pituitary. However, in order to check the functionality of the Hypothalamic Pituitary Adrenal (HPA) Axis the entire axis must be tested by way of ACTH stimulation test, CRH stimulation test and perhaps an Insulin Tolerance Test (ITT). In order to check for Addison's Disease, the auto-immune type of primary adrenal insufficiency, labs should be drawn to check 21-hydroxylase autoantibodies. Endocrine Society Guidelines (2016) ${ }^{2}$ recommend a short corticotropin test (gold standard diagnostic test). Long corticotropin test helps to distinguish between primary and secondary hypoadrenalism. ${ }^{1-6}$

\section{Treatment Strategies}

The prevalence of primary AI is $100-140$ cases per million and the rate of incidence is nearly 4 per million annually in western societies. ${ }^{2}$ The mainstay of treatment is corticosteroid replacement therapy with careful monitoring. The recommended management strategy include:

\section{Patient education}

a. It is the key to successful treatment. Patients should be advised to carry 'steroid card' or 'alert bracelet' and never miss a dose.

b. They should be informed when to modify the dose and carry an emergency pack for self-management.

\section{Treatment of chronic primary adrenal insufficiency}

\section{Glucocorticoid replacement [4-7]}

a. Must be given to all patients after confirmed diagnosis. Hydrocortisone is the pharmaceutical congener of endogenous cortisol and most commonly used. Oral administration of 15 to $25 \mathrm{mg}$ to be given 2-3 times/day with higher morning dose (usually $1 / 2$ to $2 / 3$ ) and next dose after lunch and in afternoon. (e.g. 10-5-5mg).

b. Prednisolone can be given $3-5 \mathrm{mg} / \mathrm{d}$ in $1-2$ divided doses in patients with poor compliance, T1DM with adrenal insufficiency patients instead of dexamethasone due to risk of Cushingoid sideeffects.

c. Monitoring to be done by clinical assessment like weight, postural $\mathrm{BP}$, signs and symptoms of excess steroid levels etc. instead by hormonal measurements.

d. Regular follow up at specialist centers every 6-12 months and TSH annually. ${ }^{4}$

\section{Mineralocorticoid replacement [7-9]}

a. Once-daily fludrocortisone in $50 \mathrm{mcg}$ (for adult) starting dose without any salt restriction and reducing the dose if hypertension appears. No need to commence when hydrocortisone dose $>50$ mg per $24 \mathrm{~h}$

b. Monitoring by clinical assessment like salt-craving, serum electrolytes, postural hypotension or edema. Plasma renin activity should be within upper-third of normal limits.

\section{DHEA (Dehydroepiandrosterone) replacement}

Women with low libido, depression on replacement therapy for insufficiency can be considered for DHEA administration by single morning dose for 6 months only $(25-50 \mathrm{mg} /$ day), monitored by morning serum DHEAS and SHBG measurement.

\section{Treatment during pregnancy}

a. Pregnant patients with Primary Adrenal Insufficiency (PAI) must be monitored by clinical assessment of glucocorticoid over/under replacement during therapy.

b. Hydrocortisone dose should be increased by $25-50 \%$ during 3 rd trimester.

c. During hyperemesis-gravidarum and labour, hydrocortisone must be given parenterally along with fluid replacement (100mg IM 6 hourly upto 24-48 hours after labour).

\section{Treatment during childhood}

a. In children, hydrocortisone $\left(8 \mathrm{mg} / \mathrm{m}^{2} / \mathrm{d}\right)$ is recommended as starting dose in 3-4 divided doses and synthetic, long acting glucocorticoids are avoided.

b. Monitoring by clinical assessment like growth velocity, weight, BP etc.

c. In PAI with hypoaldosteronism, fludrocortisone should be started with $100 \mathrm{mcg} /$ day. In infants and newborns sodium chloride supplementation is recommended.

\section{Treatment of acute adrenal crisis [5]}

a. Initial resuscitation and fluid correction by normal saline is mandatory. Sodium correction to be done gradually.

b. $100 \mathrm{mg}$ hydrocortisone IV to be started followed by $200 \mathrm{mg} / 24$ hours by 6 hourly injection/continuous IV (rate of infusion is $10 \mathrm{mg}$ /hour) till oral feed started

c. The stress dose of hydrocortisone in different ages (by IM/IV):

- Neonate - 6 weeks: $25 \mathrm{mg}$ stat initially, then 5-10mg 6 hourly.

- 6 weeks -3 years: $25 \mathrm{mg}$ stat initially, then $10 \mathrm{mg} 6$ hourly.

- Children 3-12 years: 50mg stat initially, then $12.5-25 \mathrm{mg}, 6$ hourly.

- Adolescents and adults: $100 \mathrm{mg}$ stat initial dose, then $25-50 \mathrm{mg}$ 6 hourly (The Royal Children's Hospital Melbourne Clinical Practice Guideline, 2017). ${ }^{5}$

a. After oral feed has been stated, hydrocortisone is given orally in double replacement dose $(20-10-10 \mathrm{mg})$ in adults. In children usually $30-50 \mathrm{mg} / \mathrm{m}^{2} / \mathrm{d}$ in 3 divided doses is started and gradually tapered to maintenance dose $\left(10-15 \mathrm{mg} / \mathrm{m}^{2} /\right.$ day in primary and $6-8 \mathrm{mg} / \mathrm{m}^{2} /$ day in secondary adrenal insufficiency).

b. Initially no mineralocorticoid replacement is required but after oral feed started, fludrocortisones, it can be commenced at $100 \mathrm{mcg} /$ day.

c. Supportive: Glucose supplementation and treatment of hypoglycemia by dextrose, treatment of hyperkalemia (if serum potassium is $>7 \mathrm{mmol} / \mathrm{L}$ with ECG changes), treatment of infection/stress factor and monitoring by serum urea, electrolytes.

\section{Treatment of secondary adrenal insufficiency [6-9]}

a. The cause in hypothalamic-pituitary disorders is mostly tumor, therefore, evaluation and management of underlying causes to be done like in surgical cases. 
b. $15-20 \mathrm{mg}$ hydrocortisone per $24 \mathrm{~h}$ to be initiated. If there is a borderline fail in cosyntropin test, only a $10 \mathrm{mg}$ or stress dose cover is recommended.

c. Replacement of thyroid hormone without cortisol can precipitate acute adrenal insufficiency.

d. Patients with hypopituitarism with partial/total ACTH deficiency and on suboptimal cortisol therapy may develop symptoms of cortisol deficiency upon initiation of growth hormone therapy due to inhibition of 11-beta-hydroxysteroid dehydrogenase- type-1. ${ }^{6-9}$

\section{Once daily long acting hydrocortisone therapy}

Current replacement strategies require modification due to evidence of impaired health and increased mortality as result of inappropriate glucocorticoid therapy. Mah et al. ${ }^{7}$ concluded that a thrice daily regimen is preferable, but immediate release hydrocortisone with short half-life $\left(t^{1 / 2}\right)$ cannot properly replicate physiological cortisol release, so delayed-release hydrocortisone are being explored. Prednisolone and dexamethasone have longer $t^{1 / 2}$ but cause high night-time glucocorticoid activity resulting in detrimental effects on insulin sensitivity and bone mineral density. ${ }^{10}$ Therefore, there was an immense need for more physiological long acting preparations:

a. Modified release hydrocortisone (Dual release DR-HC) are being investigated in few trials. ${ }^{11}$

b. Plenadren is a modified release preparation of hydrocortisone licensed recently. It has extended release core covered by immediate release coating, administered once daily and replicated normal cortisol rhythm. It showed improvement in quality of life (QOL), blood pressure and other metabolic parameters in initial trial. $^{12}$

c. Chronocort, is another modified release formulation, but it is required in a twice daily administration. It was brought by Diurnal Limited, Cardiff, UK. It was given at $10 \mathrm{pm}$, when the midnight cortisol levels were low but peak level were reached (around $380 \mathrm{nmol} / \mathrm{L}$ ) at $6-7 \mathrm{am}$ mimicking the normal cortisol rhythm. ${ }^{13}$

d. Nilsson AG et al. ${ }^{9}$ concluded favorable results on safety and tolerance of DR-HC during 24 months of therapy. ${ }^{9}$

e. Quinker $\mathrm{M}$ et al. $^{14}$ demonstrated that modified release hydrocortisone lowers body-mass index (BMI) and glycosylated hemoglobin (HbAlc) and stabilize QOL compared to conventional therapy. ${ }^{14}$

Ongoing DREAM study is designed to compare the efficacy of plenadren with conventional therapy in adrenal insufficiency patients. $^{15}$

\section{Conclusion}

The diagnosis of AI is adequately established by the ACTH stimulation $(250 \mu \mathrm{g})$ test in most patients. Glucocorticoids provide life saving treatment, but long-term quality of life is impaired, perhaps because therapy is not given in a physiologic way. The current recommended total daily dose is lower than that often prescribed. Supraphysiologic hydrocortisone doses may aid in the reversal of septic shock independent of underlying adrenal function. AI is associated with reduced quality of life that may be caused by non-physiological glucocorticoid replacement. In critical illness, glucocorticoids may reverse hemodynamic shock independent of adrenal function but do not improve mortality. Recent, reports of HIV-associated infections and medication-induced hypocortisolism are reminders that all autoimmune adrenal destruction does not underlie all cases. Progress has been made in identifying underlying pathophysiology, including genetic basis, that predispose to the development of AI in patients, however, there exact role is still under exploration.

\section{Acknowledgments}

None.

\section{Conflicts of Interset}

None.

\section{References}

1. Carey RM. The changing clinical spectrum of adrenal insufficiency. Annals of internal medicine. 1997;127(12):1103-1105.

2. Bornstein SR, Allolio B, Arlt W, et al. Diagnosis and treatment of primary adrenal insufficiency: an endocrine society clinical practice guideline. J Clin Endocrinol Metab. 2005;101(2):364-389.

3. Debono M, Ross RJ. What is the best approach to tailoring hydrocortisone dose to meet patient needs in 2012? Clin Endocrinol (Oxf). 2013;78(5):659-664.

4. Arlt W. The approach to the adult with newly diagnosed adrenal insufficiency. J Clin Endocrinol Metab. 2009;94(4):1059-1067.

5. http://www.rch.org.au/clinicalguide/guideline_index/Adrenal_crisis_ and_acute_adrenal_insufficiency/

6. Merza Z, Rostami-Hodjegan A, Memmott A, et al. Circadian hydrocortisone infusions in patients with adrenal insufficiency and congenital adrenal hyperplasia. Clin Endocrinol (Oxf). 2006;65(1):4550 .

7. Mah PM, Jenkins RC, Rostami-Hodjegan A, et al. Weight-related dosing, timing and monitoring hydrocortisone replacement therapy in patients with adrenal insufficiency. Clin Endocrinol (Oxf). 2004;61(3):367-375.

8. Jódar E, Valdepeñas MP, Martinez G, et al. Long-term follow-up of bone mineral density in Addison's disease. Clin Endocrinol (Oxf). 2003;58(5):617-620.

9. Nilsson AG, Marelli C, Fitts D, et al. Prospective evaluation of long-term safety of dual-release hydrocortisone replacement administered once daily in patients with adrenal insufficiency. Eur $J$ Endocrinol. 2014;171(3):369-377.

10. Abdel-Moneim A, Ragab AR. Cancer Protein Metabolism: Review on Etiology, Progression and Management. European Journal of Biomedical and Pharmaceutical sciences. 2016;3(6):26-44.

11. Nilsson AG, Bergthorsdottir R, Burman P, et al. Long-term safety of once-daily, dual-release hydrocortisone in patients with adrenal insufficiency: a phase 3b, open-label, extension study. Eur J Endocrinol. 2017;176(6):715-725.

12. Grossman A, Johannsson G, Quinkler M, et al. Therapy of endocrine disease: Perspectives on the management of adrenal insufficiency: clinical insights from across Europe. Eur J Endocrinol. 2013;169(6):R165R175.

13. Wass J, Owen K. Oxford handbook of endocrinology and diabetes. (3rd edn), Oxford University Press, India, 2014. p.272-278.

14. Quinkler M, Nilsen RM, Zopf K, et al. Modified-release hydrocortisone decreases BMI and $\mathrm{HbAlc}$ in patients with primary and secondary adrenal insufficiency. Eur J Endocrinol. 2015;172(5):619-626.

15. Toledo-Corral CM, Myers SJ, Li Y, et al. Blunted nocturnal cortisol rise is associated with higher carotid artery intima-media thickness (CIMT) in overweight African American and Latino youth. Psychoneuroendocrinology. 2013;38(9):1658-1667. 